\title{
Visceral artery aneurysms: evolving interdisciplinary management and future role of the abdominal surgeon
}

\author{
Vittorio Branchi ${ }^{1}$, Carsten Meyer ${ }^{2}$, Frauke Verrel ${ }^{1}$, Alexander Kania ${ }^{1}$, Edwin Bölke ${ }^{3 *}$, Alexander Semaan ${ }^{1}$, \\ Arne Koscielny ${ }^{1}$, Jörg C. Kalff ${ }^{1}$ and Hanno Matthaei ${ }^{1}$
}

\begin{abstract}
Background: Visceral artery aneurysms (VAA) are rare vascular lesions. Clinically silent VAA are increasingly detected by cross-sectional imaging but some lesions are at risk for rupture with severe bleeding. The aim of the present study was to evaluate the trends in the interdisciplinary management at a tertiary center.

Methods: Patients who underwent treatment for VAA at University Hospital of Bonn between 2005 and 2018 were enrolled in this retrospective study. Demographic, clinical, VAA-specific data as well as information on therapy, early and long-term outcome were collected and statistically analyzed.
\end{abstract}

Results: Forty-two consecutive patients, 19 females and 23 males with a median age of 59 years (range 30-91 years), were diagnosed with 56 VAA. The majority were true aneurysms $(N=32 ; 57 \%)$, whereas $43 \%(N=24)$ were pseudoaneurysms. The most common localization was the splenic artery $(N=18 ; 32 \%)$ and the average diameter was $3 \mathrm{~cm}$ (range 1-5 cm). Twenty-five patients (59.5\%) had VAA-related symptoms such as chronic abdominal pain and hemorrhage at primary diagnosis, while the diagnosis was incidental in 17 patients (40.5\%). Eleven patients (26\%) underwent open surgery whereas 29 patients (69\%) received an endovascular treatment. Patients with pseudoaneurysms were significantly older $(P=0.003)$, suffered more often from associated symptoms $(P<0.001)$ and required more emergency interventions $(P<0.0001)$ compared to those with true VAA. In the last years, the number and proportion of true VAA increased significantly $(P<0.001)$ while a significantly larger proportion could be managed interventionally $(P=0.017)$.

Conclusions: VAA are increasingly detected on imaging with lesions presenting very heterogeneously. Due to the risk of lethal rupture and in the absence of reliable prognostic markers, all the patients with VAA should be offered definite treatment. Localization, anatomy and the end-organ perfusion after intervention or operation are the most important aspects to consider when planning a treatment for VAA. For this reason, a multidisciplinary evaluation of every individual patient is necessary for an optimized outcome.

Keywords: Visceral artery aneurysms, Hemorrhage, Emergency bleeding, Interventional radiology, Open surgery

\footnotetext{
*Correspondence: edwin.boelke@med.uni-duesseldorf.de

${ }^{3}$ Department of Radiotherapy and Radiation Oncology, Faculty of Medicine, Henirich-Heine Universität, Moorenstrasse 5 ,

40225 Düsseldorf, Germany

Full list of author information is available at the end of the article
} 


\section{Introduction}

Visceral artery aneurysms (VAA) are rare yet serious vascular lesions. Their incidence has been rising over the last decades, largely due to the demographic shift and the wide-spread use of cross-sectional imaging [1, $2]$. VAA may arise in every splanchnic artery and are either classified into true aneurysms (TVAA) or pseudoaneurysms (PVAA) [3]. True aneurysms consist of abnormal arterial wall dilatation of more than 1.5 times its normal diameter involving all three vascular layers [3]. Pseudoaneurysms are characterized by disruption of the media and intimal layers of the arterial wall, resulting in a confined hematoma with connection to the arterial lumen [4]. VAA etiopathogenesis is still not completely understood. Risk factors include collagen-related diseases such as Ehlers-Danlos syndrome or non-atherosclerotic, non-inflammatory diseases of the blood vessels like fibromuscular dysplasia $[5,6]$. Interestingly, atherosclerosis seems to play only a marginal pathogenic role in aneurysm genesis [7]. The most common VAA site is the splenic artery, with a relative incidence of about $60 \%$, followed by hepatic artery aneurysms representing approximately $20 \%$ [8-10]. Nowadays, VAA are increasingly diagnosed as incidental findings on CT, MRI or angiography imaging studies [11, 12]. Hemodynamic instability may occur due to intraabdominal bleeding in the event of VAA perforation, which is associated with a high mortality of $20-100 \%$ [12, 13].

Most symptomatic VAA patients presenting to the emergency room complain about abdominal pain and are, therefore, initially triaged for surgical evaluation. In case of severe intraabdominal hemorrhage due to perforation, an immediate emergency laparotomy might be inevitable to control the bleeding. Therefore, basic skills in vascular surgery are necessary for every surgeon performing emergency explorations in a hemodynamic instable patient. In fact, conventional surgery has been the standard of care in symptomatic as well as asymptomatic VAA for decades. Development and constant improvement of minimal invasive endovascular treatment have enriched the therapeutic repertoire for VAAs but also changed the therapeutic approach drastically. However, VAA are heterogeneous and a therapeutic approach has to be individualized for every patient by a multidisciplinary team including abdominal and vascular surgeons as well as interventional radiologists $[3,11]$. The aim of this study was to evaluate the evolving interdisciplinary treatment of VAA at our tertiary center.

\section{Methods}

\section{Patients}

Patients with VAA diagnosis (ICD I72.8) admitted between 08/2005 and 08/2018 at the University Hospital of Bonn were included in this unicentric retrospective study. Ethical standards of the University of Bonn were fully acknowledged. Every patient signed informed consent before any kind of procedure. Patients with renal artery aneurysms were excluded. Elective treatment decisions were discussed for hemodynamically stable patients in a weekly interdisciplinary vascular board. In the event of VAA rupture with acute bleeding, interdisciplinary consultation was performed in the emergency room to decide for the most appropriate immediate treatment. The interdisciplinary team in both elective and emergency settings always included an abdominal surgeon, a vascular surgeon as well as an interventional radiologist. Pseudonymized data on patients, surgical and interventional therapy as well as on VAA characteristics were collected and analyzed. For the latter, an experienced diagnostic and interventional radiologist (CM) reviewed all relevant imaging studies and precisely measured and reclassified each VAA. To identify a possible change of treatment over the entire study period from 2005-2018, two subgroups were defined comprising patients presenting before ("early" group) and after January 1st, 2013 ("recent” group). The cutoff was chosen arbitrarily. Data concerning early outcomes (i.e., minor and major perioperative/ interventional complications, hospital stay, and 30-day mortality) as well as long-term performance (i.e., overall survival and disease-related chronic morbidities) were reviewed. Complications were ranked according to the Clavien-Dindo classification [14].

\section{Statistics}

Descriptive statistics (i.e., median, range, mean and standard deviation). Chi square test or $T$ test was used where appropriate. Analyses were performed with the software SPSS Statistics (IBM, Armonk, New York, USA). A $P<0.05$ was considered as statistically significant.

\section{Results \\ Patients and outcome}

During the study period, a total of 56 VAA in 42 consecutive patients including 19 females and 23 males with a median age of 59 years (range 30-91 years) were diagnosed with VAA. Detailed data are shown in Table 1. Of 
Table 1 Patients and VAA characteristics according to the clinical presentation

\begin{tabular}{|c|c|c|c|c|}
\hline \multirow[t]{2}{*}{$\mathrm{N}$ of patients } & \multirow{2}{*}{$\begin{array}{l}\text { Total } \\
42\end{array}$} & \multirow{2}{*}{$\begin{array}{l}\text { SVAA } \\
25\end{array}$} & \multirow{2}{*}{$\begin{array}{l}\text { IVAA } \\
17\end{array}$} & \\
\hline & & & & \\
\hline Age median (range) & $59(30-91)$ & $63(30-91)$ & $58(41-74)$ & $P=0.32$ \\
\hline Female $n(\%)$ & $19(45 \%)$ & $9(36 \%)$ & $10(59 \%)$ & $P=0.15$ \\
\hline Male $n(\%)$ & $23(55 \%)$ & $16(64 \%)$ & $7(41 \%)$ & \\
\hline Diameter in $\mathrm{cm}$ mean (SD) & $3( \pm 2.1)$ & $2.5( \pm 1.5)$ & $3.6( \pm 2.5)$ & $P=0.37$ \\
\hline Symptoms $n(\%)$ & $25(59.5 \%)$ & $25(100 \%)$ & $0(0 \%)$ & \\
\hline Abdominal pain $n(\%)$ & $8(19 \%)$ & $8(32 \%)$ & $0(0 \%)$ & \\
\hline Intraabdominal hemorrhage $n(\%)$ & $17(40.5 \%)$ & $17(68 \%)$ & $0(0 \%)$ & \\
\hline $\mathrm{N}$ of aneurysms & 56 & 34 & 22 & \\
\hline Splenic artery n (\%) & $18(32 \%)$ & $7(21 \%)$ & $11(50 \%)$ & $P=0.14$ \\
\hline Hepatic artery $n(\%)$ & $13(23 \%)$ & $10(29 \%)$ & $3(14 \%)$ & \\
\hline Superior mesenteric artery $n(\%)$ & $9(16 \%)$ & $5(15 \%)$ & $4(18 \%)$ & \\
\hline Gastroduodenalis artery $n(\%)$ & $9(16 \%)$ & $7(21 \%)$ & $2(9 \%)$ & \\
\hline Left gastric artery $n(\%)$ & $4(7 \%)$ & $2(6 \%)$ & $2(9 \%)$ & \\
\hline Celiac trunk $n(\%)$ & $3(5 \%)$ & $3(9 \%)$ & $0(0 \%)$ & \\
\hline $\mathrm{N}$ of true aneurysms & $32(57 \%)$ & $13(38 \%)$ & $19(86 \%)$ & $P=0.0002$ \\
\hline N of pseudoaneurysms & $21(37.5 \%)$ & $20(59 \%)$ & $1(4 \%)$ & \\
\hline Indetermined morphology & $3(5 \%)$ & $1(3 \%)$ & $2(9 \%)$ & \\
\hline Operation $n(\%)$ & $11(26 \%)$ & $5(20 \%)$ & $6(35 \%)$ & $P=0.25$ \\
\hline Intervention n (\%) & $29(69 \%)$ & 19 (76\%) & $10(59 \%)$ & \\
\hline Coiling $n(\%)$ & $23(54.5 \%)$ & $14(56 \%)$ & $9(53 \%)$ & $P=0.28$ \\
\hline Stenting $n(\%)$ & $4(9.5 \%)$ & $4(16 \%)$ & $0(0 \%)$ & \\
\hline Stenting + coiling $n(\%)$ & $2(5 \%)$ & $1(4 \%)$ & $1(6 \%)$ & \\
\hline No operation/intervention n (\%) & $2(5 \%)$ & $1(4 \%)$ & $1(6 \%)$ & $P=0.76$ \\
\hline Complications $n(\%)$ & $17(40.5 \%)$ & $11(44 \%)$ & $6(35 \%)$ & $P=0.68$ \\
\hline Dindo 1 & $2(5 \%)$ & $0(0 \%)$ & $2(12 \%)$ & $P=0.15$ \\
\hline Dindo 2 & $8(19 \%)$ & $5(20 \%)$ & $3(18 \%)$ & \\
\hline Dindo 3 & $5(12 \%)$ & $4(16 \%)$ & $1(6 \%)$ & \\
\hline Dindo 4 & $2(5 \%)$ & $2(8 \%)$ & $0(0 \%)$ & \\
\hline Median hospital stay in days (range) & $15(1-238)$ & $18(1-238)$ & $15(2-43)$ & $P=0.14$ \\
\hline $\mathrm{N}$ of deceased patients & $9(21 \%)$ & $8(32 \%)$ & $1(6 \%)$ & $P=0.043$ \\
\hline Median follow-up in months (range) & $10.5(0-151)$ & $11(0-151)$ & $10(1-130)$ & $P=0.28$ \\
\hline
\end{tabular}

IVAA incidental visceral artery aneurysm, SVAA symptomatic visceral artery aneurism, SD standard deviation

Italic values indicate statistical significance $(P<0.05)$

those, 17 Patients (40\%) had an acute abdominal hemorrhage at the time of initial diagnosis, and 8 patients $(19 \%)$ had a history of chronic abdominal pain. While 34 patients had a solitary VAA, 8 patients had multiple synchronous VAA in up to 5 different localizations. Median VAA size was $3 \mathrm{~cm}$ (range $1-5 \mathrm{~cm}$ ). The most common VAA location was the splenic artery $(N=18 ; 32 \%)$, followed by hepatic artery aneurysms $(N=13 ; 23 \%)$ (Fig. 1$)$. A total of 11 patients (26\%) underwent an open surgical operation, whereas 29 patients $(69 \%)$ were treated with an endovascular procedure.

In particular, aneurysmorrhaphy was performed in four patients (9.5\%); aneurysm resection with autologous vein patch reconstruction was performed in three patients
(7\%); two patients underwent splenectomy (5\%) and aneurysm resection with end-to-end anastomosis and aneurysm resection with synthetic graft interposition was performed in one patient (2\%), respectively. Among the endovascular procedures, coiling was performed in 23 patients (54.5\%), stenting in 4 patients (9.5\%), and a combination of stenting and coiling in 2 patients $(5 \%)$ (Figs. 2 and 3).

Complication rate was $40.5 \%(N=17)$ mostly including Clavien-Dindo Grade 2 adverse events. The median hospital stay was 15 days (range 1-238 days). Median followup was 10.5 months (0-151 months) and nine patients died (21\%). Only one patient died in strict association of his VAA (2\%). 


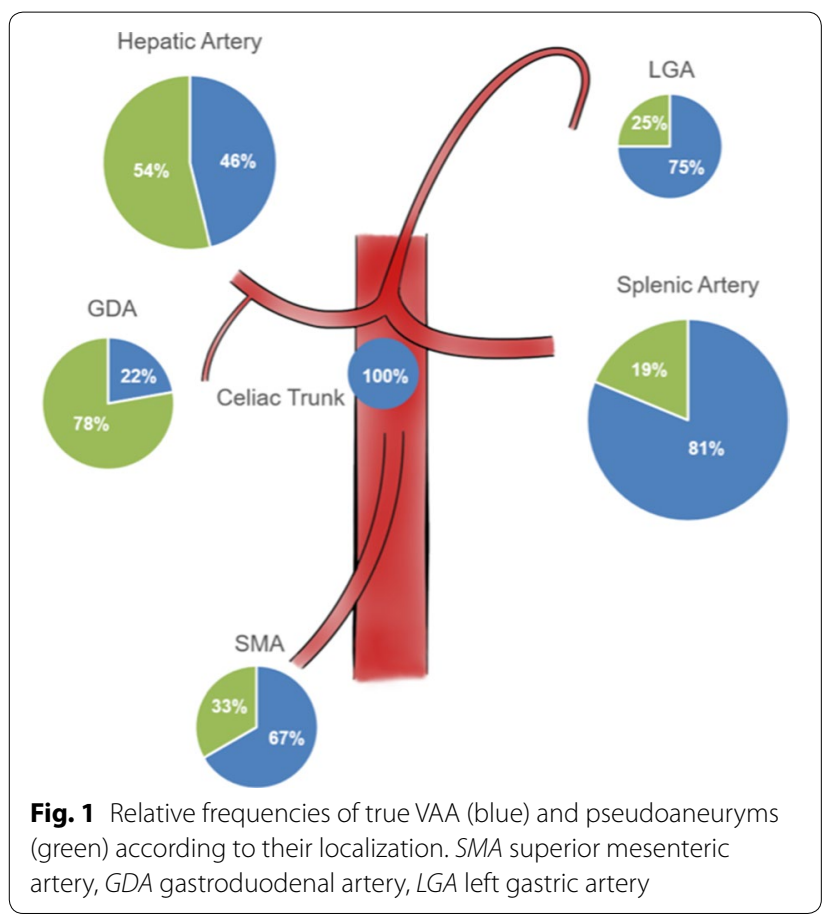

\section{Conservative management of two VAA patients}

Until 2012, every patient diagnosed with VAA has been treated either surgically or interventionally. Since 2013, two patients $(5 \%)$ with treatment indication did not receive any VAA-specific therapy. The first patient had a $3.1-\mathrm{cm}$ aneurysm of the hepatic artery and a $3.6-\mathrm{cm}$ aneurysm of the left gastric artery, both incidentally detected. The patient refused any kind of intervention and only presented to the first follow-up at 2 months after primary diagnosis, when the aneurysms size and morphology proved stable. Thereafter, the patient refused any further follow-up evaluations. The second patient had a 2-cm aneurysm of the celiac trunk and a $1.3-\mathrm{cm}$ aneurysm of the superior mesenteric artery both of which were asymptomatic. A radiological intervention was contraindicated due to the site and anatomy of the aneurysms, and the patient refused surgery. During 37 months of regular follow-up (every 2 months for the first year, every 4 months during the second year and twice a year thereafter), both VAA remained stable and asymptomatic.

\section{Asymptomatic versus symptomatic VAA}

A total of 17 patients (40\%) had no history of VAArelated symptoms and were incidentally detected (IVAA) while 25 patients $(60 \%)$ had VAA-related symptoms at the time of primary diagnosis (SVAA). PVAA were more frequently found in the SVAA group $(N=20 ; 59 \%$ vs. $N=1 ; 4 \% ; P=0.0002)$ and these patients had a higher mortality $(N=8 ; 32 \%$ vs. $N=1 ; 6 \% ; P=0.043)$. Both groups were similar, however, regarding the parameters age, gender, maximum diameter of VAA, type of intervention, complication rate and duration of hospital stay.

\section{True aneurysms versus pseudoaneurysms}

Among the 56 VAA, a majority of $57 \%$ were true aneurysms (TVAA: $N=32$ ), whereas $37 \%$ were pseudoaneurysms (PVAA: $N=17$ ). Three VAA could not be assigned to either group based on radiological or pathological assessments. Characteristics are summarized in Table 2. Patients with PVAA were significantly older
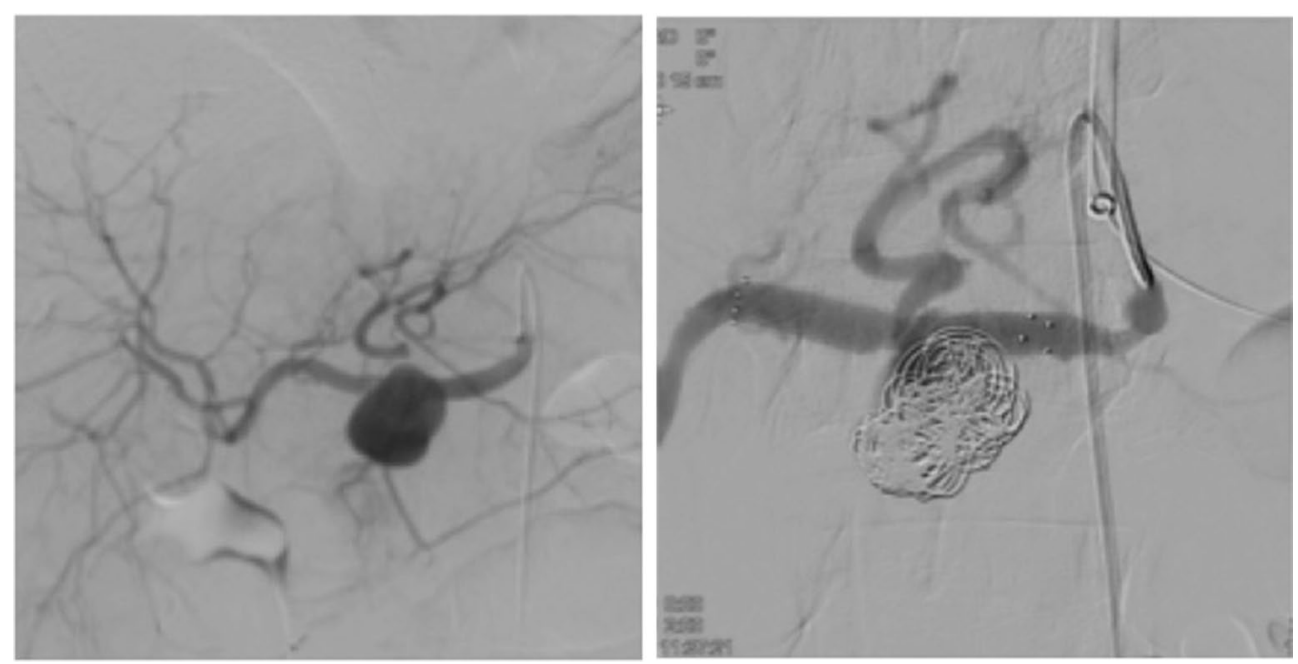

Fig. 2 A 3.5-cm aneurysm of the hepatic artery before (left) and after (right) a combined radiological intervention with stenting and coiling 

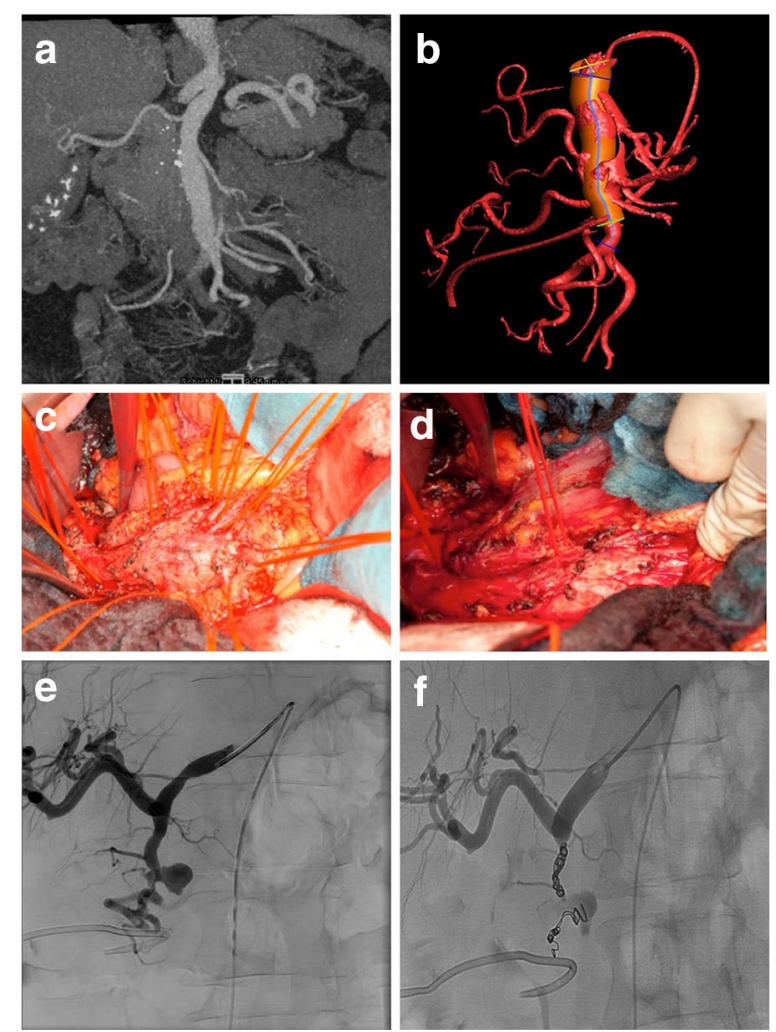

Fig. 3 CT scan of a 62-year-old patient with a big asymptomatic SMA aneurysm (a). Digital reconstruction of the CT scan in $\mathbf{a}(\mathbf{b})$. Intraoperative images of the same patient before (c) and (d) after isolation of 14 collateral branches. Angiography of a 37-year-old patient with an aneurysm of the gastroduodenal artery after perforation (e). Same patient in e after a successful coiling procedure (f)

compared to patients with TVAA (median 72 years vs. 57 years; $P=0.003)$ and suffered more often from symptoms such as abdominal pain $(N=16 ; 94 \%$ vs. $N=8$; $36 \% ; P=0.0002$ ). Localization of VAA differed in both groups since TVAA were typically localized in the splenic artery $(N=13 ; 41 \%)$, whereas PVAA were mainly localized in the hepatic $(N=7 ; 33 \%)$ and gastroduodenal artery $(N=7 ; 33 \%)$. Emergency treatment was more frequently necessary in the PVAA group $(N=15 ; 88 \%$ vs. $N=1 ; 4.5 \% ; P<0.0001)$. In addition, hospital stay of these patients was significantly longer (22 days vs. 15 days; $P=0.028)$ and their mortality rate higher $(N=7 ; 41 \%$ vs. $N=2 ; 9 \% ; P=0.018)$. Both groups were, however, similar regarding the parameters gender, maximum diameter of VAA, and type of intervention.

\section{VAA treatment and outcome in the early versus recent period}

Among the 42 patients, 17 were diagnosed before (40\%) and 25 after January 1st, 2013 (60\%) (Table 3). Patients in the recent treatment group where significantly younger than those in the earlier group (median age 58 years vs. 71 years; $P=0.01$ ). Furthermore, there was a significantly higher rate of true aneurysms detected during more recent years ( $N=26 ; 76 \%$ vs. $N=6 ; 27 \% ; P=0.0006)$. In the early period, $47 \%$ of the patients underwent primary surgery for VAA $(N=8)$, whereas $53 \%$ were treated with an endovascular procedure $(N=9)$. More recently, however, a vast majority of patients were treated with radiological intervention $(N=20 ; 80 \%$ vs. $N=5 ; 20 \%)$, and this shift in therapeutic strategy proved to be statistically significant $(P=0.017)$. Patients' characteristics in the early and in the recent treatment group were, however, similar in terms of gender, clinical presentation, VAA dimensions and localization, complication rate and hospital stay.

\section{Discussion}

The use of diagnostic cross-sectional imaging has drastically increased in the last decades, particularly in highvolume cancer and emergency centers [15-17]. This, in combination with the ongoing demographic shift in Western countries appears to be responsible for the increasing incidence of VAA as previously described [18, 19]. Although this vascular disorder is undoubtedly rare, we noticed a slight increase in VAA incidence over the last 13 years, which specifically encouraged us to conduct this retrospective study.

A VAA often presents as acute abdomen with lifethreatening hemorrhage. Approximately, $40 \%$ of our patients suffered from intraabdominal bleeding, mostly in combination with hemorrhagic shock. This high rate of VAA perforation at primary diagnosis noticed in our cohort is comparable to previously described incidence rates $[1,2,11,20]$. Our data furthermore confirmed that especially pseudoaneurysms carry an extremely high risk for rupture. This might be related to their wall instability compared to true VAA. We observed pseudoaneurysms occasionally after major abdominal surgery such as hepatobiliary and pancreatic tumor resections. This dangerous condition tends to occur in elderly patients in the first postoperative days. In particular, pseudoaneurysms can develop after pancreatic leakage or pancreatitis because of enzymatic digestion of the arterial wall while any "index bleeding" from abdominal drains should be considered for immediate contrast-enhanced CT scan.

Due to the potentially fatal consequences of VAA perforation, there has been an effort to identify reliable risk factors predicting aneurysm growth and rupture. Undoubtedly, posttraumatic pseudoaneurysms require instant treatment to avoid severe morbidity and lethal courses. In contrast to pseudoaneurysm, the natural history of true VAA and their possible progression over 
Table 2 Patients and VAA characteristics according to the aneurysm morphology

\begin{tabular}{|c|c|c|c|}
\hline $\mathrm{N}$ of patients & True VAA & Pseudo VAA & \\
\hline & 22 & 17 & \\
\hline Age median (range) & $57(30-79)$ & $72(36-91)$ & $P=0.003$ \\
\hline Female $n(\%)$ & $12(45.5 \%)$ & $11(65 \%)$ & $P=0.52$ \\
\hline Male $n(\%)$ & $10(55.5 \%)$ & $6(35 \%)$ & \\
\hline Diameter in cm mean (SD) & $3( \pm 1.6)$ & $2( \pm 1.3)$ & $P=0.10$ \\
\hline Symptoms $n(\%)$ & $8(36 \%)$ & $16(94 \%)$ & $P=0.0002$ \\
\hline Abdominal pain $n(\%)$ & $7(31 \%)$ & $1(6 \%)$ & $P<0.0001$ \\
\hline Intraabdominal hemorrhage $n(\%)$ & $1(5 \%)$ & $15(88 \%)$ & \\
\hline $\mathrm{N}$ of aneurysms & 32 & 21 & \\
\hline Splenic artery $n(\%)$ & $13(41 \%)$ & $3(14 \%)$ & $P=0.046$ \\
\hline Hepatic artery n (\%) & $6(19 \%)$ & $7(33 \%)$ & \\
\hline Superior mesenteric artery $n(\%)$ & $6(19 \%)$ & $3(14 \%)$ & \\
\hline Left gastric artery $n(\%)$ & $3(9 \%)$ & $1(5 \%)$ & \\
\hline Gastroduodenalis artery $n(\%)$ & $2(6 \%)$ & $7(33 \%)$ & \\
\hline Celiac trunk $n(\%)$ & $2(6 \%)$ & $0(0 \%)$ & \\
\hline Emergency $n(\%)$ & $1(4.5 \%)$ & $15(88 \%)$ & $P<0.0001$ \\
\hline Elective $n(\%)$ & $21(95 \%)$ & $2(12 \%)$ & \\
\hline Operation n (\%) & $5(23 \%)$ & $3(18 \%)$ & $P=0.58$ \\
\hline Intervention n (\%) & $15(68 \%)$ & $14(82 \%)$ & \\
\hline Coiling $n(\%)$ & $13(59 \%)$ & $10(59 \%)$ & $P=0.51$ \\
\hline Stenting $n(\%)$ & $1(4.5 \%)$ & $3(18 \%)$ & \\
\hline Stenting + coiling $n(\%)$ & $1(4.5 \%)$ & $1(6 \%)$ & \\
\hline No operation/intervention n (\%) & $2(9 \%)$ & $0(0 \%)$ & $P=0.20$ \\
\hline Complications $n(\%)$ & $8(36 \%)$ & $7(41 \%)$ & $P=0.073$ \\
\hline Dindo $1 n(\%)$ & $2(9 \% \%)$ & $0(0 \%)$ & $P=0.25$ \\
\hline Dindo $2 n(\%)$ & $3(14 \%)$ & $3(18 \%)$ & \\
\hline Dindo $3 n(\%)$ & $3(14 \%)$ & $2(12 \%)$ & \\
\hline Dindo $4 n(\%)$ & $0(0 \%)$ & $2(12 \%)$ & \\
\hline Median hospital stay in days (range) & $15(2-43)$ & $22(1-238)$ & $P=0.0028$ \\
\hline Median follow-up in months (range) & $13(1-112)$ & $2(0-59)$ & $P=0.054$ \\
\hline $\mathrm{N}$ of deceased patients & $2(9 \%)$ & $7(41 \%)$ & $P=0.018$ \\
\hline
\end{tabular}

Three VAA could not be assigned to either group based on radiological or pathological assessments. VAA visceral artery aneurysm, $S D$ standard deviation Italic values indicate statistical significance $(P<0.05)$

time is hard to predict. Hence, there is an ongoing debate about indication and the right timing of therapy especially in the absence of VAA-related symptoms. Due to small retrospective studies and the complete absence of prospective randomized trial, the evidence level regarding VAA treatment is low. Nevertheless, pregnancy is the only established risk factor for rupture in patients with true splenic aneurysms. Aneurysms of this location, more frequent in females (4:1), are even four times more common in multiparous women owing to hormonerelated vascular changes [21, 22]. For this reason, it has been proposed that all incidentally detected aneurysms of the splenic artery during pregnancy, or even in women of childbearing age, should be promptly treated [23, 24]. Aside from VAA type and location, there has been an ongoing debate about size of true VAA with respect to timing of treatment. According to several expert opinions, all true aneurysm $>2 \mathrm{~cm}$ in maximum diameter must be treated $[4,25,26]$, which is in accordance with the guidelines defined by the German Association for Vascular Surgery (Deutsche Gesellschaft für Gefäßchirurgie, DGG) [27]. However, due their unpredictable growth, therapy of every VAA irrespective of their diameter is encouraged according to DGG recommendations. Furthermore, an endovascular approach should be aimed for. Still, an emergency laparotomy needs to be performed in case of VAA rupture or if an interventional procedure does not appear to be a safe option or is unavailable. In selected patients, a watchful waiting approach may occasionally be justified. For example, in the case of 
Table 3 Patients and VAA characteristics according to the period of treatment

\begin{tabular}{|c|c|c|c|}
\hline $\mathrm{N}$ of patients & Before 2013 & Since 2013 & \\
\hline & 17 & 25 & \\
\hline Age median (range) & $71(47-91)$ & $58(30-80)$ & $P=0.01$ \\
\hline Female $n(\%)$ & $6(35 \%)$ & $13(52 \%)$ & $P=0.29$ \\
\hline Male $n(\%)$ & $11(65 \%)$ & $12(48 \%)$ & \\
\hline Diameter in $\mathrm{cm}$ mean (SD) & $2.9( \pm 1.5)$ & $2.6( \pm 1.5)$ & $P=0.52$ \\
\hline Symptoms $n(\%)$ & $11(65 \%)$ & $14(41 \%)$ & $P=0.57$ \\
\hline Abdominal pain $n(\%)$ & $3(18 \%)$ & $5(15 \%)$ & $P=0.65$ \\
\hline Intraabdominal hemorrhage $n(\%)$ & $8(47 \%)$ & $9(26 \%)$ & \\
\hline $\mathrm{N}$ of aneurysms & 22 & 34 & \\
\hline Splenic artery $n(\%)$ & $5(23 \%)$ & $13(38 \%)$ & $P=0.76$ \\
\hline Hepatic artery $n(\%)$ & $6(27 \%)$ & $7(21 \%)$ & \\
\hline Superior mesenteric artery $n(\%)$ & $5(23 \%)$ & $4(12 \%)$ & \\
\hline Gastroduodenalis artery $n(\%)$ & $3(14 \%)$ & $6(18 \%)$ & \\
\hline Left gastric artery $n(\%)$ & $2(9 \%)$ & $2(6 \%)$ & \\
\hline Celiac trunk $n(\%)$ & $1(5 \%)$ & $2(6 \%)$ & \\
\hline $\mathrm{N}$ of true aneurysms & $6(27 \%)$ & $26(76 \%)$ & $P=0.0006$ \\
\hline $\mathrm{N}$ of pseudoaneurysms & $13(59 \%)$ & $8(24 \%)$ & \\
\hline Indetermined morphology & $3(14 \%)$ & $0(0 \%)$ & \\
\hline Operation $n(\%)$ & $8(47 \%)$ & $3(12 \%)$ & $P=0.017$ \\
\hline Intervention $n(\%)$ & $9(53 \%)$ & $20(80 \%)$ & \\
\hline Coiling $n(\%)$ & $7(41 \%)$ & $16(64 \%)$ & $P=0.45$ \\
\hline Stenting $n(\%)$ & $2(12 \%)$ & $2(8 \%)$ & \\
\hline Stenting + coiling $n(\%)$ & $0(0 \%)$ & $2(8 \%)$ & \\
\hline No operation/intervention $n(\%)$ & $0(0 \%)$ & $2(8 \%)$ & $P=0.23$ \\
\hline Emergency & $8(47 \%)$ & $9(36 \%)$ & $P=0.62$ \\
\hline Elective & $9(53 \%)$ & $14(56 \%)$ & \\
\hline Complications $n(\%)$ & $9(53 \%)$ & $8(32 \%)$ & $P=0.17$ \\
\hline Dindo 1 & $0(0 \%)$ & $2(8 \%)$ & $P=0.25$ \\
\hline Dindo 2 & $6(35 \%)$ & $2(8 \%)$ & \\
\hline Dindo 3 & $2(12 \%)$ & $3(12 \%)$ & \\
\hline Dindo 4 & $1(6 \%)$ & $1(4 \%)$ & \\
\hline Median hospital stay in days (range) & $16(1-238)$ & $13(2-122)$ & $P=0.61$ \\
\hline Median follow-up in months (range) & $37(0-151)$ & $7(0-46)$ & $P=0.0007$ \\
\hline $\mathrm{N}$ of deceased patients & $2(12 \%)$ & $7(28 \%)$ & $P=0.21$ \\
\hline
\end{tabular}

$V A A$ visceral artery aneurysm, $S D$ standard deviation

Italic values indicate statistical significance $(P<0.05)$

severe comorbidities and morphologically stable, small TVAA, a conservative treatment may be considered.

There is a constant ambition to improve both surgical and interventional techniques to ensure the least invasive procedure for every VAA detected. As reported, in recent years a shift has been noticed towards a therapeutic paradigm prioritizing radiological intervention over surgery. The question of whether VAA should be managed by open surgery or endovascular treatment has never been addressed by randomized studies, and only small retrospective cohorts have been published.
Cochennec et al. [28] compared open and endovascular repairs in two European institutions over a 15-year period in a retrospective study. Sixteen patients were treated by open repair, and 15 patients by endovascular procedures. The authors noticed no significant difference between open repair and endovascular therapy in terms of 30-day mortality rate and perioperative complications. Marone et al. [29] compared surgical and endovascular treatment in a cohort of 94 consecutive patients. Sachdev et al. [30] compared 24 patients with surgical repair to 35 who underwent endovascular treatment. The authors 
reported an $89 \%$ success rate following coil embolization or stent-graft therapy confirming the efficacy of endovascular treatment for VAA, which was comparable to results in the surgical cohort. Paralleling these findings, the data from our center are encouraging with respect to both the safety and the efficacy of radiological procedures thereby reaching success rates after surgery with a tendency to shorter hospital stays. Despite these findings, the surgeon will continue to play a central role in the management of complicated VAA reflected by $25 \%$ of all and $12 \%$ of recent VAA patients needing open surgery. Aside from hemorrhage control and basic vascular reconstruction, the abdominal surgeon should critically and constantly evaluate the end-organ perfusion. In fact, in case of inadequate vascular supply, the abdominal surgeon should promptly perform procedures such as bowel resections, splenectomy or partial liver resections.

\section{Implications, perspective and limitations of the study}

The aim of the present study was to evaluate the trend in the interdisciplinary VAA management at a tertiary center. We retrospectively analyzed a consecutive series of 42 VAA patients over a period of more than 10 years. One of the main practical contributions of the present research is that it underlines the shift toward an endovascular therapeutic paradigm during the last few years with excellent short- and long-term outcomes. In addition, this study highlighted the substantial differences between PVAA and TVAA in terms of outcome. In fact, symptomatic VAA are mainly PVAA and incidental VAA are mainly TVAA. This should help an interdisciplinary team through the decision process to identify high-risk patients, therefore, orienting toward an early elective intervention.

Our study has some limitations. Inherent to its retrospective design, we cannot draw valid conclusions regarding optimal treatment of true VAA and pseudoaneurysms. Ideally, prospective multicenter randomized trials are required to clearly define the optimal treatment for VAA. However, it is questionable whether a conservative treatment arm can be ethically justified due to the high morbidity and mortality associated with VAA perforation and other VAA-related complications such as endorgan ischemia.

\section{Conclusion}

VAA is a rare vascular disease. Nonetheless, it should be considered as a differential diagnosis in the event of acute abdomen, in particular in the presence of hemodynamic instability. Abdominal and vascular surgeons should be aware of the therapeutic options in case an endovascular procedure is contraindicated or unavailable. Abdominal surgeons should be prepared to handle both, emergencies involving VAA rupture and also complicated cases, in which an intervention does not represent the definitive therapy. A multidisciplinary evaluation is the key to an appropriate treatment allocation in this heterogeneous disease. An evaluation at a tertiary center with high expertise in visceral and vascular surgery and interventional radiology is mandatory. A multi-institutional registry and, at best, prospective trials might help improve diagnostic strategies and identify prognostic factors to eventually establish evidence-based guidelines for VAA management.

\section{Abbreviations \\ VAA: visceral artery aneurysms; TVAA: true aneurysms; PVAA: pseudoaneu- rysms; IVAA: incidental visceral artery aneurysms; SVAA: symptomatic visceral artery aneurysms; CT: computed tomography; MRI: magnetic resonance imag- ing; DGG: Deutsche Gesellschaft für Gefäßchirurgie-German Association for Vascular Surgery.}

\section{Authors' contributions}

$\mathrm{VB}$ and $\mathrm{HM}$ contributed to study conception and design. VB and AK acquired the data. $V B, A S, C M$ and $A K$ analyzed and interpreted the data. $V B, E B$ and $H M$ drafted the manuscript. JCK, AK, EB and FV contributed to the discussion and the data interpretation and critical revised the manuscript. All the authors read and approved the final manuscript.

\section{Author details \\ 1 Department of General, Visceral, Thoracic and Vascular Surgery, University Hospital Bonn, Sigmund-Freud-Strasse 25, 53127 Bonn, Germany. ${ }^{2}$ Depart- ment of Radiology, University Hospital Bonn, Sigmund-Freud-Strasse 25, 53127 Bonn, Germany. ${ }^{3}$ Department of Radiotherapy and Radiation Oncology, Faculty of Medicine, Henirich-Heine Universität, Moorenstrasse 5, 40225 Düsseldorf, Germany.}

\section{Competing interests}

The authors declare that they have no competing interests.

Availability of data and materials

The datasets generated and analyzed are available from the corresponding author on reasonable request.

\section{Consent for publication \\ Not applicable.}

\section{Ethics approval and consent to participate}

All the procedures performed in studies involving human participants were in accordance with the ethical standards of the institutional and/or national research committee and with the 1964 Helsinki Declaration and its later amendments or comparable ethical standards. For this type of study, formal consent is not required.

\section{Funding}

No sources of funding were used for this study.

\section{Publisher's Note}

Springer Nature remains neutral with regard to jurisdictional claims in published maps and institutional affiliations.

Received: 19 November 2018 Accepted: 14 February 2019

Published online: 28 February 2019 


\section{References}

1. Chadha M, Ahuja C. Visceral artery aneurysms: diagnosis and percutaneous management. Semin Intervent Radiol. 2009;26:196-206.

2. Gehlen JMLG, Heeren PAM, Verhagen PF, et al. Visceral artery aneurysms. Vasc Endovascular Surg. 2011:45:681-7.

3. Chiaradia M, Novelli L, Deux JF, et al. Ruptured visceral artery aneurysms. Diagn Interv Imaging. 2015;96:797-806.

4. Pasha S, Gloviczki P, Stanson A, et al. Splanchnic artery aneurysms. Mayo Clin Proc. 2007:82:472-9.

5. Slovut DP, Olin JW. Fibromuscular dysplasia. N Engl J Med. 2004;350:1862-71.

6. Shalhub S, Black JH, Cecchi AC, et al. Molecular diagnosis in vascular Ehlers-Danlos syndrome predicts pattern of arterial involvement and outcomes. J Vasc Surg. 2014;60:160-9.

7. Pulli R, Dorigo W, Troisi N, et al. Surgical treatment of visceral artery aneurysms: a 25-year experience. J Vasc Surg. 2008:48:334-42.

8. Chiesa R, Astore D, Guzzo G, et al. Visceral artery aneurysms. Ann Vasc Surg. 2005:19:42-8.

9. Ikeda O, Tamura Y, Nakasone Y, et al. Nonoperative management of unruptured visceral artery aneurysms: treatment by transcatheter coil embolization. J Vasc Surg. 2008;47:1212-9.

10. Jesinger RA, Thoreson AA, Lamba R. Abdominal and pelvic aneurysms and pseudoaneurysms: imaging review with clinical, radiologic, and treatment correlation. Radiographics. 2013;33:E71-96.

11. Carr SC, Mahvi DM, Hoch JR, et al. Visceral artery aneurysm rupture. JVasc Surg. 2001;33:806-11.

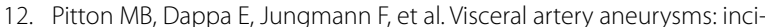
dence, management, and outcome analysis in a tertiary care center over one decade. Eur Radiol. 2015:25:2004-14.

13. Schweigert M, Adamus R, Stadlhuber RJ, et al. Endovascular stent-graft repair of a symptomatic superior mesenteric artery aneurysm. Ann Vasc Surg. 2011;25(841):e5-8.

14. Dindo D, Demartines N, Clavien P-A. Classification of surgical complications: a new proposal with evaluation in a cohort of 6336 patients and results of a survey. Ann Surg. 2004;240:205-13.

15. Bhargavan M, Sunshine J. Utilization of radiology services in the United States: levels and trends in modalities, regions, and populations. Radiology. 2005;234:824-32.

16. Mills AM, Raja AS, Marin JR. Optimizing diagnostic imaging in the emergency department angela. Acad Emerg Med. 2015;22:625-31.
17. Dinan MA, Curtis LH, Hammill BG, et al. Changes in the use and costs of diagnostic imaging among medicare beneficiaries with cancer, 1999-2006. JAMA. 2010;303:1625-31.

18. Tulsyan N, Kashyap VS, Greenberg RK, et al. The endovascular management of visceral artery aneurysms and pseudoaneurysms. J Vasc Surg. 2007;45:276-83.

19. Ferrero E, Viazzo A, Ferri M, et al. Management and urgent repair of ruptured visceral artery aneurysms. Ann Vasc Surg. 2011;25(981):e7-11.

20. Huang $\mathrm{Y}, \mathrm{H}$ sieh $\mathrm{H}$, Tsai $\mathrm{F}$, et al. Visceral artery aneurysm: risk factor analysis and therapeutic opinion. Eur J Vasc Endovascular Surg. 2007;301:293-301.

21. Dave SP, Reis ED, Hossain A, et al. Splenic artery aneurysm in the 1990s. Ann Vasc Surg. 2000;14:223-9.

22. Meyer A, Uder M, Lang W, et al. Visceral artery aneurysms. Zentralbl Chir. 2010;135:416-20.

23. Abbas MA, Stone WM, Fowl RJ, et al. Splenic artery aneurysms: two decades experience at Mayo clinic. Ann Vasc Surg. 2002;16:442-9.

24. Ha JF, Phillips M, Faulkner K. Splenic artery aneurysm rupture in pregnancy. Eur J Obstet Gynecol Reprod Biol. 2009:146:133-7.

25. Cordova AC, Sumpio BE. Visceral artery aneurysms and pseudoaneurysms - should they all be managed by endovascular techniques? Ann Vasc Dis. 2013;6:687-93.

26. Belli AM, Markose G, Morgan R. The role of interventional radiology in the management of abdominal visceral artery aneurysms. Cardiovasc Intervent Radiol. 2012;35:234-43.

27. DGG. Diagnostik und Therapie der Aneurysmen des Truncus coeliacus, $\operatorname{der}$ A. lienalis, hepatica und mesenterica. http://www.gefässchirurgie.de/ LL Viiszeralarterienaneurysmen_2011.pdf. Accessed 28 Aug 2018.

28. Cochennec F, Riga CV, Allaire E, et al. Contemporary management of splanchnic and renal artery aneurysms: results of endovascular compared with open surgery from two European vascular centers. Eur J Vasc Endovasc Surg. 2011:42:340-6.

29. Marone EM, Mascia D, Kahlberg A, et al. Is open repair still the gold standard in visceral artery aneurysm management? Ann Vasc Surg. 2011;25:936-46.

30. Sachdev U, Baril DT, Ellozy SH, et al. Management of aneurysms involving branches of the celiac and superior mesenteric arteries: a comparison of surgical and endovascular therapy. J Vasc Surg. 2006;44:718-24.
Ready to submit your research? Choose BMC and benefit from:

- fast, convenient online submission

- thorough peer review by experienced researchers in your field

- rapid publication on acceptance

- support for research data, including large and complex data types

- gold Open Access which fosters wider collaboration and increased citations

- maximum visibility for your research: over $100 \mathrm{M}$ website views per year

At BMC, research is always in progress.

Learn more biomedcentral.com/submissions 